\title{
A Partial Differential Equation with Infinitely many Periodic Orbits: Chaotic Oscillations of a Forced Beam
}

\author{
Philip Holmes * \\ Department of Mathematics \\ Arizona State University, Tempe, AZ 85287 \\ Jerrold E. Marsden ${ }^{\dagger}$ \\ Department of Mathematics \\ University of California, Berkeley, CA 94720, USA
}

1981. This version: July 20, 1994

\begin{abstract}
This paper delineates a class of time-periodically perturved evolution equations in a Banach space whose associated Poincaré map contains a Smale horseshoe. This implies that such systems possess periodic orbits with arbitrarily high period. The method uses techniques originally due to Melnikov and applies to systems of the form $\dot{x}=f_{0}(x)+\varepsilon f_{1}(x, t)$, where $\dot{x}=f_{0}(x)$ is Hamiltonian and has a homoclinic orbit. We give an example from structural mechanics: sinusoidally forced vibrations of a buckled beam.
\end{abstract}

\section{Introduction: A Physical Model}

In this paper we give sufficient conditions on $T$-periodically forced evolution equations in a Banach space for the existence of a Smale horseshoe for the time- $T$ map of the dynamics. This implies the existence of infinitely many periodic orbits of arbitrarily high period and suggests the existence of a strange attractor. The results here are an extension of infinite dimensions of some of those in Holmes [1979a,b, 1980a] and Chow, Hale and Mallet-Paret [1980].

The techniques used are invariant methods, nonlinear semigroups and an extension to infinite dimensions of Melnikov's method [1963] for planar ordinary differential equations. The results are applied to the equations of a nonlinear, periodically forced, buckled beam. As the external force is increased, we show that a global bifurcation occurs, resulting in the transversal intersection of stable and unstable manifolds. This leads to all the complex dynamics of a horseshoe (Smale [1963]).

The study of chaotic motion in dynamical systems is now a burgeoning industry. The mechanism given here is just one of many that can lead to chaotic dynamics. For a different mechanism occuring in reaction-diffusion equations, see Guckenheimer [1979].

A physical model will help motivate the analysis. Consider a beam that is buckled by an external load $\Gamma$, so there are two stable and one unstable equilibrium state (see Figure 1). The whole structure is then shaken with a transverse periodic displacement, $f \cos \omega t$. The beam moves due to its inertia. In a related experiment (see Tseng and Dugundji [1971] and Moon and Holmes [1979], and remarks

\footnotetext{
*Research partially supported by NSF Contract DMS 89-19074 and CTS 89-06343

${ }^{\dagger}$ Research partially supported by DOE Contract DE-FGO3-92ER25129.
} 
Figure 1:

below), one observes periodic motion about either of the two stable equilibria for small $f$, but as $f$ is increased, the motion becomes aperiodic or chaotic. The mathematical problem is to provide theorems that help to explain this behavior.

There are a number of specific models that can be used to describe the beam in Figure 1. One of these is the following partial differential equation for the transverse deflection $w(z, t)$ of the centerline of the beam:

$$
\ddot{w}+w^{\prime \prime \prime \prime}+\Gamma w^{\prime \prime}-\mathcal{X}\left(\int_{0}^{1}\left[w^{\prime}\right]^{2} d \xi\right)=\varepsilon(f \cos \omega t-\delta \dot{w}) .
$$

where ${ }^{\cdot}=\partial / \partial t, \quad{ }^{\prime}=\partial / \partial z, \Gamma=$ external load, $\mathcal{X}=$ stiffness due to "membrane" effects, $\delta=$ damping, and $\varepsilon$ is a parameter used to measure the relative size of $f$ and $\delta$. Amongst many possible boundary conditions we shall choose $w=w^{\prime \prime}=0$ at $z=0,1$; i.e., simply supported, or hinged ends. With these boundary conditions, the eivenvalues of the linearized, unforced equations, i.e., complex numbers $\lambda$ such that

$$
\lambda^{2} w+w^{\prime \prime \prime \prime}+\Gamma w^{\prime \prime}=0
$$

for some non-zero $w$ satisfying $w=w^{\prime \prime}=0$ at $z=0,1$, form a countable set

$$
\lambda_{j}= \pm \pi j \sqrt{\Gamma-\pi^{2} j^{2}}, \quad j=1,2, \ldots
$$

Thus, if $\Gamma<\pi^{2}$, all eigenvalues are imaginary and the trivial solution $w=0$ is formally stable; for positive damping it is Liapunov stable. We shall henceforth assume that

$$
\pi^{2}<\Gamma<4 \pi^{2},
$$

in which case the solution $w=0$ is unstable with one positive and one negative eigenvalue and the nonlinear equation (1) with $\varepsilon=0, \mathcal{X}>0$ has two nontrivial stable buckled equilibrium states.

A simplified model for the dynamics of (1) is obtained by seeking lowest mode solutions of the form

$$
w(z, t)=x(t) \sin (\pi z) .
$$

Substitution into (1) and taking the inner product with the basis function $\sin (\pi z)$, gives a Duffing type equation for the modal displacement $x(t)$ :

$$
\ddot{x}-\beta x+\alpha x^{3}=\varepsilon(\gamma \cos \omega t-\delta \dot{x}),
$$

where $\beta=\pi^{2}\left(\Gamma-\pi^{2}\right)>0, \alpha=\mathcal{X} \pi^{4} / 2$ and $\gamma=4 f / \pi$. Equation (2) was studied at length in earlier papers (see Holmes [1979a, 1979b] and Holmes and Marsden [1979]). This work uses Melnikov's 
method; see Melnikov [1963], Arnold [1964], and Holmes [1980a]. Closely related results are obtained by Chow, Hale, and Mallet-Paret [1980]. This method allows one to estimate the separation between stable and unstable manifolds and to determine where they intersect transversally. The method given in the above references apply to periodically perturbed two-dimensional flows such as the dynamics of equation (2). In this paper we extend these ideas to infinite dimensional evolution equations on Banach spaces and apply the method to the evolution equation (1).

Tseng and Dugundji [1971] studied the one and two mode Galerkin approximation of (1) and found "chaotic snap-through" motions in numerical integrations. Such motions were found experimentally but were not studied in detail. Subsequently, Moon and Holmes [1979] found similar motions in experiments with an elastic, ferromagnetic beam and showed that a single-mode Galerkin approximation could indeed admit infinite sets of periodic motions of arbitrarily high period (Holmes $[1979 b])$.

It is known that the time $t$-maps of the Euler and Navier-Stokes equations written in Lagrangian coordinates are smooth. Thus the methods of this paper apply to these equations, in principle. On regions with no boundary, one can regard the Navier-Stokes equations with forcing as a perturbation of a Hamiltonian system (the Euler equations); see Ebin and Marsden [1970]. Thus, if one knew that a homoclinic orbit existed for the Euler equations, then the methods of this paper would produce infinitely many periodic orbits with arbitrarily high period, indicative of terbulence. No specific examples of this are known to us. One possibility, however, is periodically forced surface waves. See Gollub [1980].

An unforced sine-Gordon equation possesses heteroclinic orbits, as was shown by Levi, Hoppenstead, and Miranker [1978]. Methods of this paper were used by Holmes [1980b] to show that this system, with weak periodic forcing and damping and defined on a finite spatial domain, contain horseshoes. The methods should also be useful for travelling wave problems on infinite domains, such as the Korteweg-e Vries equation.

\section{Abstract Hypothesis}

We consider an evolution equation in a Banach space $X$ of the form

$$
\dot{x}=f_{0}(x)=\varepsilon f_{1}(x, t)
$$

where $f_{1}$ is periodic of period $T$ in $t$. Our hypotheses on (3) are as follows:

\section{Hypothesis 1}

(a) Assume $f_{0}(x)=A x+B(x)$ where $A$ is an unbounded) linear operator which generates a $C^{0}$ one parameter group of transformations on $X$ and where $B: X \rightarrow X$ is $C^{\infty}$. Assume that $B(0)=0$ and $D B(0)=0$.

(b) Assume $f_{1}: X \times S^{1} \rightarrow X$ is $C^{\infty}$ where $S^{1}=\mathbf{R} /(T)$, the circle of length $T$.

Assumption 1 implies that the associated suspended autonomous system on $X \times S^{1}$,

$$
\begin{aligned}
\dot{X} & =f_{0}(x)+\varepsilon f_{1}(x, \theta), \\
\dot{\theta} & =1,
\end{aligned}
$$

has a smooth local flow, $F_{t}^{\varepsilon}$. This means that $F_{t}^{\varepsilon}: X \times S^{1} \rightarrow X \times S^{1}$ is a smooth map defined for small $|t|$ which is jointly continuous in all variables $\varepsilon, t, x \in X, \theta \in S^{1}$ and for $x_{0}$ in the domain of $A, t \mapsto F_{t}^{\varepsilon}\left(x_{0}, \theta_{0}\right)$ is a unique solution of (4) with initial condition $x_{0}, \theta_{0}$.

This implication results from a theorem of Segal [1962]. For a simplified proof, see Holmes and Marsden [1987, Prop. 2.5] and for generalizations, see Marsden and McCracken [1976].

The final part of assumption 1 follows: 
(c) Assume that $F_{t}^{\varepsilon}$ is defined for all $t \in \mathbb{R}$ for $\varepsilon>0$ sufficiently small.

To verify this in examples, one must obtain an a priori bound on the $X$-norm of solutions of (4) to ensure they do not escape to infinity in a finite time. This is sufficient by the local existence theory alluded to above. In examples of concern to us, (c) will be verified using straightforward energy estimates. See Holmes and Marsden [1978] for related examples.

Our second assumption is that the unperturbed system is Hamiltonian. This means that $X$ carries a skew symmetric continuous bilinear map $\Omega: X \times X r i \mathbb{R}$ which is weakly non-degenerate (i.e., $\Omega(u, v)=0$ for all $v$ implies $u=0$ ) called the symplectic form and there is a smooth function $H_{0}: X \rightarrow \mathbb{R}$ such that

$$
\Omega\left(f_{0}(x), u\right)=d H_{0}(x) \cdot u
$$

for all $x$ in $D_{A}$, the domain of $A$. Consult Abraham and Marsden [1978] and Chernoff and Marsden [1974] for details about Hamiltonian systems. For example, these assumptions imply that the unperturbed system conserves energy:

$$
H_{0}\left(F_{t}^{0}(x)\right)=H_{0}(x)
$$

(For $\varepsilon=0$ we drop the dependence on $\theta$.) We summarize this condition and further restriction as follows:

(a) Assume that the unperturbed system $\dot{x}=f_{0}(x)$ is Hamiltonian with energy $H_{0}: X \rightarrow \mathbb{R}$.

(b) Assume there is a symplectic 2-manifold $\Sigma \subset X$ invariant under the flow $F_{t}^{0}$ and that on $\Sigma$ the fixed point $p_{0}=0$ has a homoclinic orbit $x_{0}(t)$, i.e.,

$$
\dot{x}_{0}(t)=f_{0}\left(x_{0}(t)\right)
$$

and

$$
\lim _{t=+\infty} x_{0}(t)=\lim _{t \rightarrow-\infty} x_{0}(t)=0
$$

Remarks on Assumption 2.

(a) For a non-Hamiltonian two-dimensional version, see Holmes [1980a] and Chow, Hale, and Mallet-Paret [1980]. Non-Hamiltonian infinite dimensional analogues could probably be developed by using the methods of this paper.

(b) The condition that $\Sigma$ be symplectic means that $\Omega$ restricted to vectors tangent to $\Sigma$ defines a non-degenerate bilinear form. We also note that by a general theorem of Chernoff and Marsden [1974], the restriction of $F_{t}^{0}$ to $\Sigma$ is generated by a smooth vector field on $\Sigma$; i.e., the dynamics within $\Sigma$ is governed by ordinary differential eqiations. The situation described in assumption 2 is illustrated in Figure 2(a).

(c) Assumption 2 can be replaced by a similar assumption on the existence of heteroclinic orbits connecting two saddle poitns and the existence of transverse heteroclinic orbits can then be proven using the methods below. For details in the two-dimensional case, see Holmes [1980a]. Theorems guaranteeing the existence of saddle connections may be found in Conley and Smoller [1974] and Koppel and Howard [1979].

(d) To apply the techniques that follow, one must be able to calculate $x_{0}(t)$ either explicitly or numerically. In our examples, we find it analytically; for numerical methods, see Hassard [1980]. 
Figure 2:

Next we introduce a non-resonance hypothesis.

(H3)

(a) Assume that the forcing term $f_{1}(x, t)$ in (3) has the form

$$
f_{1}(x, t)=A_{1} x+f(t)+g(x, t)
$$

where $A_{1}: X \rightarrow X$ is a bounded linear operator, $f$ is periodic with period $T, g(x, t)$ is t-periodic with period $T$ and satisfies $g(0, t)=0, D_{x} g(0, t)=0$, so $g$ admits the estimate

$$
\|g(x, t)\| \leq(\text { Const })\|x\|^{2}
$$

for $x$ in a neighborhood of 0.

(b) Suppose that the "linearized" system

$$
\dot{x}_{L}=A x_{L}+\varepsilon A_{1} x_{L}+\varepsilon f(t)
$$

has a T-periodic solution $x_{\lambda}(t, \varepsilon)$ such that $x_{L}(t, \varepsilon)=O(\varepsilon)$.

Remarks on (H3)

1. For finite dimensional systems, (H3) can be replaced by the assumption that 1 does not lie in the spectrum of $e^{T A}$; i.e. none of the eigenvalues of $A$ resonate with the forcing frequency.

2. For the beam problem, with $f(t)=f(z) \cos \omega t$, (b) means that $\omega \neq \pm \lambda_{n}, n=1,2, \ldots$, where $i \lambda_{n}$ are the purely imaginary eigenvalues of $A$. This is seen by solving the component forced linear oscillator equations. As we shall see, more delicate non-resonance requirements would be necessary for general (smooth) $T$-periodic perturbations, not of the form (5).

3. For the beam problem we can take $g=0$. We have included it in the abstract theory for use in other examples such as the sine-Gordon equation.

Next, we need an assumption that $A_{1}$ contributes positive damping and that $p_{0}=0$ is a saddle. 
(a) For $\varepsilon=0, e^{T A}$ has a spectrum consisting in two simple real eigenvalues $e^{ \pm \lambda T}, \lambda \neq 0$, with the rest of the spectrum on the unit circle.

(b) For $\varepsilon>0, e^{T\left(A+\varepsilon A_{1}\right)}$ has a spectrum consisting in two simple real eigenvalues $e^{T \lambda_{\varepsilon}^{ \pm}}$varying continuously in $\varepsilon$ from perturbation theory; cf. Kato [1977]) with the rest of the spectrum, $\sigma_{R}^{\varepsilon}$, inside the unit circle $|z|=1$ and obeying the estimates

$$
C_{2} \varepsilon \leq \operatorname{distance}\left(\sigma_{R}^{\varepsilon},|z|=1\right) \leq C_{1} \varepsilon
$$

for $C_{1}, C_{2}$ positive constants.

Remarks on (H4).

1. In general it can be awkward to estimate the spectrum of $e^{T A}$ in terms of the spectrum of A. Some information is contained in Hille and Phillips [1957] and Carr [1980]. See also Carr and Malhardeen [1980], Vidav [1970], Shizuta [1979] and Rauch [1979]. For the beam problem with $\varepsilon=0$ it is sufficient to use these two facts or a direct calculation:

(a) If $A$ is skew adjoint, then $\sigma\left(e^{t A}\right)=\operatorname{closureof} e^{t \sigma(a)}$;

(b) if $X=X_{1} \oplus X_{2}$, where $X_{2}$ is finite dimensional (the eigenspace of the real eigenvalues in the beam problem) and $B_{1}$ is skew adjoint on $X_{1}$ and $B_{2}: X_{2} \rightarrow X_{2}$ is a (bounded) linear operator, then

$$
\sigma\left(e^{t\left(B_{1} \oplus B_{2}\right)}\right)=\operatorname{closure}\left(e^{\sigma t B_{1}} \cup e^{t \sigma\left(B_{2}\right)} .\right.
$$

For $\varepsilon<0$ the abstract theorems are not very helpful. In the beam example the eigenfunctions of $A+\varepsilon A_{1}$ can be computed explicitly and form a basis for $X$, so the estimates (8) can be done directly; in fact $\sigma_{R}^{\varepsilon}$ consists of a circle a distance $O(\varepsilon)$ inside the unit circle; see Appendix A.

2. The estimate $\operatorname{dist}\left(\sigma_{R}^{\varepsilon},|z|=1\right) \geq C_{2} \varepsilon$ guarantees that

$$
L_{\varepsilon}=I d-e^{T\left(A+\varepsilon A_{1}\right)}
$$

is invertible and

$$
\left\|L_{\varepsilon}^{-1}\right\| \leq \mathrm{const} / \varepsilon .
$$

3. The estimate $\operatorname{dist}\left(\sigma_{R}^{\varepsilon},|z|=1\right) \leq C_{1} \varepsilon$ guarantees that the eigenvalue $\exp \left(T \lambda_{\epsilon}^{-}\right)$will be the closest to the origin for $\varepsilon$ small. This is needed below for the existence of an invariant manifold corresponding to $\lambda_{\varepsilon}^{-}$.

Finally, we need an extra hypothesis on the nonlinear term. We have already assumed $B$ vanishes at least quadratically, as does $g$. Now we assume $B$ vanishes cubically.

(H5) $B(0)=0, D B(0)=0$ and $D^{2} B(0)=0$.

This means that in a neighborhood of 0 ,

$$
\|B(x)\| \leq \text { Const }\|x\|^{3} .
$$

(Actually $B(x)=o\left(\|x\|^{2}\right)$ would do).

Remarks on (H5). 
1. The necessity of having $B$ vanish cubically is due to the possibility of the spectrum of $A$ accumulating at zero. If this can be excluded for other easons, (H5) can be dropped and (H4) simplified. There is a similar phenomenon for ordinary differential equations noted by Jack Hale. Namely, if the linear system

$$
\frac{d}{d t}\left(\begin{array}{l}
x \\
\dot{x} \\
y
\end{array}\right)=\left(\begin{array}{c}
\dot{x} \\
x \\
-\varepsilon y
\end{array}\right)
$$

is perturbed by nonlinear terms plus forcing, to guarantee that the trivial solution $(0,0,0)$ perturbs to a periodic solution as in lemma 1 below, one needs the nonlinear terms to be $o(|x|+|\dot{x}|+|y|)^{3}$.

2. For nonlinear wave equations, positivity of energy may force $D^{2} B(0)=0$.

Consider the suspended system (4) with its flow $F_{t}^{\varepsilon}: X \times S^{1} \rightarrow X \times S^{1}$. Let $P^{\varepsilon}: X \rightarrow X$ be defined by

$$
P^{\varepsilon}(x)=\pi_{1} \cdot\left(F_{T}^{\varepsilon}(x, 0)\right)
$$

where $\pi_{1}: X \times S^{1} \rightarrow X$ is the projection onto the first factor. The map $P^{\varepsilon}$ is just the Poincaré map for the flow $F_{t}^{\varepsilon}$. Note that $P^{0}\left(p_{0}\right)=p_{0}$, and that fixed points of $P^{\varepsilon}$ correspond to periodic orbits of $F_{t}^{\varepsilon}$.

Lemma 2.1 For $\varepsilon<0$ small, there is a unique fixed point $p_{\varepsilon}$ of $P^{\varepsilon}$ near $p_{0}=0$; moreover $p_{\varepsilon}-p_{0}=$ $O(\varepsilon)$, i.e. there is a constant $K$ such that $\left\|p_{\varepsilon}\right\| \leq K \varepsilon$ for all (small) $\varepsilon$.

For ordinary differential equations, lemma 1 is a standard fact about persistence of fixed points, assuming 1 does not line in the spectrum of $e^{T A}$ (i.e. $p_{0}$ is hyperbolic). For general partial differential equations, the validity of lemma 1 can be a delicate matter. In our context of smoth perturbations of linear systems with assumptions (H1)-(H5), the result is proved in Appendic A, along with the following.

Lemma 2.2 For $\varepsilon>0$ sufficiently small, the spectrum of $D P^{\varepsilon}\left(p_{\varepsilon}\right)$ lies strictly inside the unit circle with the exception of the single real eigenvalue $e^{T \lambda_{\varepsilon}^{+}}>1$.

In lemma 1 we saw the fixed point $p_{0}$ perturbs to another fixed point $p_{\varepsilon}$ for the perturbed system. The same is true for the invariant manifolds; see Figure 2(b):

Lemma 2.3 Correspoinding to the eigenvalues $e^{T \lambda_{\varepsilon}^{ \pm}}$there are unique invariant manifolds $W^{s s}\left(p_{\varepsilon}\right)$ (the strong stale manifold) and $W^{u}\left(p_{\varepsilon}\right)$ (the unstable nmanifold) of $p_{\varepsilon}$ for the map $P^{\varepsilon}$ such that

i. $W^{s s}\left(p_{\varepsilon}\right)$ and $W^{u}\left(p_{\varepsilon}\right)$ are tangent to the eigenspace of $e^{T \lambda_{\varepsilon}^{ \pm}}$respectively at $p_{\varepsilon}$;

ii. they are invariant under $P^{\varepsilon}$;

iii. if $x \in W^{s s}\left(p_{\varepsilon}\right)$, then

$$
\lim _{n \rightarrow \infty}\left(P^{\varepsilon}\right)^{n}(x)=p_{\varepsilon}
$$

and if $x \in W^{u}\left(p_{\varepsilon}\right)$ then

$$
\lim _{n \rightarrow-\infty}\left(P^{\varepsilon}\right)^{n}(n)=p_{\varepsilon} .
$$

iv. For any finite $t^{*}, W^{s s}\left(p_{\varepsilon}\right)$ is $C^{r}$ close as $\varepsilon \rightarrow 0$ to the homoclinic orbit $x_{0}(t), t^{*} \leq t<\infty$ and for any finite $t_{*}, W^{u}\left(p_{\varepsilon}\right)$ is $C^{r}$ close to $x_{0}(t),-\infty<t \leq t_{*}$ as $\varepsilon \rightarrow 0$. Here, $r$ is any fixed integer, $0 \leq r<\infty$. 
This lemma follows from the invariant manifold theorems (Hirsh, Pugh, and Shug [1977] and the smoothness of the flow of equations (4), discussed in Appendix A.

The Poincaré map $P^{\varepsilon}$ was associated with the section $X \times\{0\}$ in $X \times S^{1}$. Equally well, we can take the section $X \times\left\{t_{0}\right\}$ to get Poincaré maps $P_{t_{0}}^{\varepsilon}$. By definition,

\section{Hamiltonian normal forms: the case $m=2$ (elliptical in- stability)}

\subsection{Basic amplitude equations}

We next consider the case in which the azimuthal wavenumbers differ by 2 . This is the case of the so-called elliptic instability studied by Pierrehumbert [1986] and Bayly [1986]. It arises when the rotation symmetry $S O(2)$ is broken to $Z_{2}$, for example, by distorting the circular streamlines of the flow into elliptical shape. This distortion couples modes propagating in the positive and negative directions along the axis. In systems with axial reflection symmetry both waves are simultaneously present. For the eigenfunction

$$
\psi(r, \phi, z)=\operatorname{Re}\left(A_{1} e^{i k z+i \phi}+A_{2} e^{-i k z+i \phi}\right),
$$

the rotations $\phi \rightarrow \phi+\theta$ act by $\left(A_{1}, A_{2}\right) \rightarrow e^{i \theta}\left(A_{1}, A_{2}\right)$, while the translations $z \rightarrow z+d$ act by $\left(A_{1}, A_{2}\right) \rightarrow\left(e^{i k d} A_{1}, e^{-i k d} A_{2}\right)$ and reflection $z \rightarrow-z$ acts by $\left(A_{1}, A_{2}\right) \rightarrow\left(A_{2}, A_{1}\right)$. As a consequence of the reflection symmetry the dispersion curves cross on the real axis, i.e., at $\omega=0$. The elliptical distortion breaks the rotation invariance and couples the two counter-propagating modes. The amplitude equations, truncated at third order, take the form

$$
\begin{gathered}
\frac{d A_{1}}{d t}=i \lambda A_{1}+\epsilon \bar{A}_{2}+i A_{1}\left(s_{1}\left|A_{1}\right|^{2}+s_{2}\left|A_{2}\right|^{2}\right) \\
\frac{d A_{2}}{d t}=i \lambda A_{2}+\epsilon \bar{A}_{1}+i A_{2}\left(s_{2}\left|A_{1}\right|^{2}+s_{1}\left|A_{2}\right|^{2}\right),
\end{gathered}
$$

where, as before, $\lambda$ is the detuning and $\epsilon$ measures the size of the elliptical distortion. The coefficient describing the coupling to the distortion can be made purely real as in (11), or purely imaginary. In either case the origin is unstable in the wedge $|\lambda|<\epsilon$, i.e.,, the reflection symmetry $z \rightarrow-z$ forces splitting to take place. This observation is independent of any detailed considerations of the system of interest. In the dissipative case equations (11) were studied by Riecke et al. [1988] (see also Walgraef [1988]), in the context of parametric forcing of the Hopf bifurcation with $O(2)$ symmetry. Riecke et al. showed that the forcing stabilized standing waves over traveling waves, even in cases where, in the absence of forcing, traveling waves would be stable. Equations (11) thus represent the nondissipative limit of the analysis of Riecke et al.

With the change of variables $A_{1}(t)=B_{1}(t) \exp (i \psi(t)), A_{2}(t)=B_{2}(t) \exp (-i \psi(t))$, where $\psi(t)=$ $-s_{2} \int_{t_{0}}^{t}\left(\left|B_{1}\right|^{2}-\left|B_{2}\right|^{2}\right) d s$, one obtains

$$
\begin{aligned}
& \frac{d B_{1}}{d t}=i \lambda B_{1}+\epsilon \bar{B}_{2}+i\left(s_{1}+s_{2}\right) B_{1}\left|B_{1}\right|^{2} \\
& \frac{d B_{2}}{d t}=i \lambda B_{2}+\epsilon \bar{B}_{1}+i\left(s_{1}+s_{2}\right) B_{2}\left|B_{2}\right|^{2}
\end{aligned}
$$


or, rescaling $B_{1}$ and $B_{2}$ :

$$
\begin{gathered}
\frac{d B_{1}}{d t}=i \lambda B_{1}+\epsilon \bar{B}_{2}+i B_{1}\left|B_{1}\right|^{2} \\
\frac{d B_{2}}{d t}=i \lambda B_{2}+\epsilon \bar{B}_{1}+i B_{2}\left|B_{2}\right|^{2} .
\end{gathered}
$$

This is the required normal form for the elliptical instability, provided $s_{1}+s_{2} \neq 0$. Here $\lambda$ is the detuning parameter and $\epsilon$ measures the size of the elliptical distortion (e.g. eccentricity). It is remarkable that the normal form (13) does not contain any parameters depending on a specific problem under consideration.

Equations (13) are Hamiltonian in the standard structure for the complex variables $z_{1}=B_{1}$ and $z_{2}=B_{2}$ with the Hamiltonian

$$
H\left(B_{1}, B_{2}\right)=-\frac{\lambda}{2}\left(\left|B_{1}\right|^{2}+\left|B_{2}\right|^{2}\right)+\epsilon \operatorname{Im}\left(B_{1} B_{2}\right)-\frac{1}{4}\left(\left|B_{1}\right|^{4}+\left|B_{2}\right|^{4}\right) .
$$

Moreover, the symmetry $\left(B_{1}, B_{2}\right) \mapsto\left(e^{i \theta} B_{1}, e^{-i \theta} B_{2}\right)$ gives the conserved quantity (momentum map)

$$
J\left(B_{1}, B_{2}\right)=\left|B_{1}\right|^{2}-\left|B_{2}\right|^{2} .
$$

As in $\S 2$, we can express this Hamiltonian structure in either polar coordinates or in terms of invariants. In terms of the variables $B_{1} \equiv r_{1} \exp \left(i \phi_{1}\right), B_{2} \equiv r_{2} \exp \left(i \phi_{2}\right)$ and $\phi \equiv \phi_{1}+\phi_{2}$, equations (13) become

$$
\begin{aligned}
\frac{d r_{1}}{d t} & =\epsilon r_{2} \cos \phi \\
\frac{d r_{2}}{d t} & =\epsilon r_{1} \cos \phi \\
\frac{d \phi}{d t} & =2 \lambda-\epsilon\left(\frac{r_{2}}{r_{1}}+\frac{r_{1}}{r_{2}}\right) \sin \phi+r_{1}^{2}+r_{2}^{2} .
\end{aligned}
$$

Again, this system is completely integrable with the two integrals derived from the above Hamiltonian (or its negative) and momentum:

$$
J=r_{1}^{2}-r_{2}^{2}, \quad E=\frac{\lambda}{2}\left(r_{1}^{2}+r_{2}^{2}\right)-\epsilon r_{1} r_{2} \sin \phi+\frac{1}{4}\left(r_{1}^{4}+r_{2}^{4}\right) .
$$

Using the integrals $J$ and $E$, the solution of (16) reduces to quadrature:

$$
\frac{1}{4}\left(\frac{d \rho}{d t}\right)^{2}=P(\rho) \equiv \epsilon^{2}\left(\rho^{2}-J^{2}\right)-4\left(E-\frac{\lambda \rho}{2}-\frac{1}{8}\left(\rho^{2}+J^{2}\right)\right)^{2} .
$$

Here $\rho \equiv r_{1}^{2}+r_{2}^{2}$. Note that for fixed $J$ the integral $E$ varies between $h_{1}(J)$ and $h_{2}(J)$, where $h_{1}(J)$ corresponds to a fixed point and $h_{2}(J)$ corresponds to a homoclinic (heteroclinic) orbit.

The system (13) has two invariant subspaces $\left\{B_{1}=B_{2}\right\}$ and $\left\{B_{1}=i B_{2}\right\}$. In the dissipative case mentioned above these correspond to the two types of oscillations phase locked to half the frequency of the parametric forcing. Both are standing oscillations. These subspaces are characterized by $J=0$. In the first subspace (13) reduce to

$$
\frac{d B}{d t}=i \lambda B+\epsilon \bar{B}+i B|B|^{2} .
$$


In the second subspace,

$$
\frac{d B}{d t}=i \lambda B-i \epsilon \bar{B}+i B|B|^{2} .
$$

The equations in each subspace are again Hamiltonian, which is consistent with the general fact that fixed point spaces of discrete symplectic symmetries are symplectic spaces and induce Hamiltonian subsystems on them (see Marsden [1992, Chapter 8] for the general theory). The corresponding phase portraits of (19) are shown in Figure 4. The phase portraits of (20) are obtained by rotation of the phase portraits in Figure 4 by $\frac{\pi}{2}$.

More generally, the phase portraits depend on both $E$ and $J$. These quantities specify invariant surfaces in phase space. Dynamics on these surfaces can be understood by converting (18) to a system of two equations in the variables $\rho$ and $\frac{d \rho}{d t}$. For $\lambda=0$ there are two possibilities, depending on the sign of $\epsilon^{2}+E-\frac{J^{2}}{8}$. The resulting phase portraits in $\left(\rho, \frac{d \rho}{d t}\right)$ space resemble those shown in Figure 4 .

\subsection{The effect of symmetry breaking}

The discussion of the elliptical instability presented above relies on the presence of reflection symmetry in the axial direction. This residual symmetry need not be exact, however, and may be broken for example by means of an axial flow. A number of other system symmetry breaking perturbations can also be envisaged. These include the following:

(a) $S O(2) \times O(2) \rightarrow Z_{2} \times O(2) \rightarrow Z_{2} \times S O(2)$

(b) $S O(2) \times O(2) \rightarrow Z_{3} \times O(2)$

(c) $S O(2) \times O(2) \rightarrow Z_{4} \times O(2)$.

In those cases where the axial reflection symmetry is preserved, the elliptical instability remains a steady state one. In cases where it is broken (for example, by axial flow) the instability becomes a Hopf bifurcation (cf. Armbruster and Mahalov [1992], Knobloch [1992b]). In the following we suppose that $\epsilon_{1}$ measures the strength of the system symmetry breaking from $S O(2)$ to $Z_{n}$, and $\epsilon_{2}$ measures the strength of the system symmetry breaking from $O(2)$ to $S O(2)$, and retain as before only the dominant symmetry breaking terms.

When an axial flow reduces the symmetry from $S O(2) \times O(2)$ to $S O(2) \times S O(2)$ by breaking the reflection symmetry $z \rightarrow-z$ the symmetry $\left(B_{1}, B_{2}\right) \rightarrow\left(B_{2}, B_{1}\right)$ of the normal form (13) is broken. Consequently we can describe the effect of weak axial flow by breaking the latter symmetry. We obtain

$$
\begin{aligned}
& \frac{d B_{1}}{d t}=i \lambda_{1} B_{1}+\epsilon_{1} p_{1} \bar{B}_{2}+i\left(1+\gamma_{1}\right) B_{1}\left|B_{1}\right|^{2} \\
& \frac{d B_{2}}{d t}=i \lambda_{2} B_{2}+\epsilon_{1} p_{2} \bar{B}_{1}+i\left(1+\gamma_{2}\right) B_{2}\left|B_{2}\right|^{2} .
\end{aligned}
$$

Here, $\lambda_{2}-\lambda_{1}=O\left(\epsilon_{2}\right), p_{2}-p_{1}=O\left(\epsilon_{2}\right)$ and $\gamma_{2}-\gamma_{1}=O\left(\epsilon_{2}\right)$, where $\epsilon_{2}$ denotes the strength of the reflection symmetry breaking effect. Note that by rescaling the amplitudes the coefficients $\gamma_{1}$ and $\gamma_{2}$ can be set equal to zero, though at the cost of redefining $p_{1}$ and $p_{2}$. It is now easy to check that the trivial equilibrium is unstable whenever

$$
\left(\lambda_{1}-\lambda_{2}\right)^{2}<4\left(\epsilon_{1}^{2} p_{1} p_{2}-\lambda_{1} \lambda_{2}\right) .
$$


Note that, in contrast to the symmetric case, the presence of the instability depends critically on the splitting of the frequencies of the left-handed and right-handed modes. If this splitting is large enough for a given value of $\epsilon$ the instability can be suppressed entirely.

Equations (21) are also integrable, and have the following two integrals:

$$
J=p_{2} r_{1}^{2}-p_{1} r_{2}^{2}, \quad E=\frac{1}{2}\left(\frac{\lambda_{1}+\lambda_{2}}{p_{1}+p_{2}}\right)\left(r_{1}^{2}+r_{2}^{2}\right)-\epsilon_{1} r_{1} r_{2} \sin \phi+\frac{1+\gamma_{1}}{4 p_{1}} r_{1}^{4}+\frac{1+\gamma_{2}}{4 p_{2}} r_{2}^{4} .
$$

The use of the integrals reduces the system (21) to quadrature:

$$
\frac{1}{4 p_{1} p_{2}}\left(\frac{d \rho}{d t}\right)^{2}=P(\rho)
$$

where $\rho \equiv p_{2} r_{1}^{2}+p_{1} r_{2}^{2}$ and

$$
\begin{aligned}
P(\rho) & \equiv \epsilon_{1}^{2}\left(\rho^{2}-J^{2}\right)-4 p_{1} p_{2}\left[E-\frac{\lambda_{1}+\lambda_{2}}{4 p_{1} p_{2}}\left(\rho+\frac{p_{1}-p_{2}}{p_{1}+p_{2}} J\right)\right. \\
& -\frac{1}{16 p_{1}^{2} p_{2}^{2}}\left[\left(\left(1+\gamma_{1}\right) p_{1}+\left(1+\gamma_{2}\right) p_{2}\right)\left(\rho^{2}+J^{2}\right)+2\left(\left(1+\gamma_{1}\right) p_{1}\right.\right. \\
& \left.\left.\left.-\left(1+\gamma_{2}\right) p_{2}\right) \rho J\right]\right]^{2} .
\end{aligned}
$$

Equations (13) and (21) can be put in rigid body form. We start with the equations

$$
\begin{aligned}
& \frac{d A_{1}}{d t}=i \lambda_{1} A_{1}+\epsilon_{1} p_{1} \bar{A}_{2}+i A_{1}\left(s_{11}\left|A_{1}\right|^{2}+s_{12}\left|A_{2}\right|^{2}\right) \\
& \frac{d A_{2}}{d t}=i \lambda_{2} A_{2}+\epsilon_{1} p_{2} \bar{A}_{1}+i A_{2}\left(s_{21}\left|A_{1}\right|^{2}+s_{22}\left|A_{2}\right|^{2}\right),
\end{aligned}
$$

describing the effect of weak axial flow on the elliptic instability (case (a) above). Here the amplitudes $A_{1}$ and $A_{2}$ are as in (10), and $\lambda_{1}-\lambda_{2}, p_{1}-p_{2}, s_{11}-s_{22}$ and $s_{12}-s_{21}$ are all $O\left(\epsilon_{2}\right)$. There are two cases: (i) $p_{1} p_{2}<0$ (pinched spheres, passing of the zero eigenvalues), and (ii) $p_{1} p_{2}>0$ (pinched hyperboloids, splitting of the zero eigenvalues). In case (ii), a rescaling of the amplitudes yields

$$
\begin{aligned}
& \frac{d A_{1}}{d t}=i \lambda_{1} A_{1}+\epsilon_{1} p \bar{A}_{2}+i A_{1}\left(s_{11}\left|A_{1}\right|^{2}+s_{12}\left|A_{2}\right|^{2}\right) \\
& \frac{d A_{2}}{d t}=i \lambda_{2} A_{2}+\epsilon_{1} p \bar{A}_{1}+i A_{2}\left(s_{21}\left|A_{1}\right|^{2}+s_{22}\left|A_{2}\right|^{2}\right) .
\end{aligned}
$$

In terms of the Euler variables

$$
N \equiv\left|A_{1}\right|^{2}+\left|A_{2}\right|^{2}, \quad w \equiv\left|A_{1}\right|^{2}-\left|A_{2}\right|^{2}, \quad u+i v=2 A_{1} A_{2},
$$

cf. equations (??), one obtains the equations

$$
\begin{aligned}
& \frac{d w}{d t}=0 \\
& \frac{d N}{d t}=2 \epsilon_{1} p u
\end{aligned}
$$




$$
\begin{aligned}
& \frac{d u}{d t}=-v\left(2 \lambda+a N+\epsilon_{2} b w\right)+2 \epsilon_{1} p N \\
& \frac{d v}{d t}=u\left(2 \lambda+a N+\epsilon_{2} b w\right),
\end{aligned}
$$

where $2 \lambda \equiv \lambda_{1}+\lambda_{2}, 2 a \equiv s_{11}+s_{21}+s_{12}+s_{22}$ and $2 \epsilon_{2} b \equiv s_{11}+s_{21}-s_{12}-s_{22}$. Note that once again $N^{2}=u^{2}+v^{2}+w^{2}$. If we replace $\epsilon_{1} p$ by $\epsilon_{1}$ and $\epsilon_{2} b$ by $\epsilon_{2}$ the vector $L \equiv(N, i u, i v)$ satisfies the top equation

$$
\frac{d L}{d t}=L \times \Omega
$$

where $\Omega \equiv P+D L$, and $P$ and $D$ are now given by

$$
P=\left(\begin{array}{c}
-2 \lambda-\epsilon_{2} w \\
0 \\
-2 i \epsilon_{1}
\end{array}\right), \quad D=\left(\begin{array}{ccc}
-a & 0 & 0 \\
0 & 0 & 0 \\
0 & 0 & 0
\end{array}\right)
$$

cf. equation (??). As in the case of the $m=1$ instability, the kinetic energy is given by $T=$ $L^{T} P+\frac{1}{2} L^{T} D L$ and is an integral of the motion. It is now easy to understand how the Hamiltonian structure (and phase portraits) change when the two system symmetry breaking parameters $\epsilon_{1}$ and $\epsilon_{2}$ are varied.

To understand case (b) we note, following Nagata [1988], that the normal form for a vector field that commutes with the symmetry $Z_{3} \times O(2)$ is given by

$$
\begin{aligned}
& \frac{d A_{1}}{d t}=i g_{1} A_{1}+i \epsilon_{1} g_{2} \bar{A}_{1}^{2} \bar{A}_{2}^{3} \\
& \frac{d A_{2}}{d t}=i g_{3} A_{2}+i \epsilon_{1} g_{4} \bar{A}_{2}^{2} \bar{A}_{1}^{3},
\end{aligned}
$$

where the functions $g_{1}, \ldots, g_{4}$ are $C^{\infty}$ functions of the invariants $\left|A_{1}\right|^{2}+\left|A_{2}\right|^{2},\left(A_{1} A_{2}\right)^{3},\left(\bar{A}_{1} \bar{A}_{2}\right)^{3}$ and $\left(\left|A_{1}\right|^{2}-\left|A_{2}\right|^{2}\right)^{2}$. These functions are not independent since the two equations for $A_{1}$ and $A_{2}$ are related by the reflection symmetry $\left(A_{1}, A_{2}\right) \rightarrow\left(A_{2}, A_{1}\right)$.

Similarly, in case (c), one finds that the normal form that commutes with the required action of $Z_{4} \times O(2)$ is

$$
\begin{aligned}
& \frac{d A_{1}}{d t}=i g_{1} A_{1}+i \epsilon_{1} g_{2} \bar{A}_{1} \bar{A}_{2}^{2} \\
& \frac{d A_{2}}{d t}=i g_{3} A_{2}+i \epsilon_{1} g_{4} \bar{A}_{2} \bar{A}_{1}^{2},
\end{aligned}
$$

where the functions $g_{1}, \ldots, g_{4}$ are now functions of the invariants $\left|A_{1}\right|^{2}+\left|A_{2}\right|^{2},\left(A_{1} A_{2}\right)^{2},\left(\bar{A}_{1} \bar{A}_{2}\right)^{2}$ and $\left(\left|A_{1}\right|^{2}-\left|A_{2}\right|^{2}\right)^{2}$. As before these functions are related by the reflection symmetry $\left(A_{1}, A_{2}\right) \rightarrow$ $\left(A_{2}, A_{1}\right)$.

It is important to observe that the symmetry breaking terms that now enter are all nonlinear and hence they do not affect the linear stability problem. Consequently in these cases instability will not in general be present. This is because the wavenumbers of the eigenfunctions differ by 2 . However, when $S O(2)$ is broken to $Z_{2 n}(n \geq 1)$ the same equations as $(27)$ are obtained for the 
interaction of modes of the form $e^{i k z+i n \phi}$ and $e^{-i k z+i n \phi}$. Similarly, when $m_{1}-m_{2}=2 n-1$, say, equations of the form (??) or (??) follow for modes of the form $e^{i k z}$ and $e^{i k z+i(2 n-1) \phi}$ whenever the symmetry is broken to $Z_{2 n-1}$, depending on the symmetry in the axial direction. Here $n$ is again a positive integer.

Equations (11) discussed above also arise in the theory of edge waves excited by a normally incident wave at a beach; equations (21) then describe the excitation of edge waves by slightly oblique waves (cf. Miles [1991]). This problem is closely related to that discussed in $\S 4$.

\section{The general case}

In this section we consider the interaction of two modes with azimuthal wavenumbers $m$ and $n$, and the same axial wavenumber. We must now distinguish between two types of parametric interaction, through coupling to a deformation mode of the form $e^{ \pm i(m-n) \phi}$ or of the form $e^{ \pm i(m+n) \phi}$, where $0<n<m$. We write the linear eigenfunction in the form

$$
\psi(r, \phi, z)=\operatorname{Re}\left(A_{m} e^{i k z+i m \phi}+A_{n} e^{i k z+i n \phi}+A_{-m} e^{i k z-i m \phi}+A_{-n} e^{i k z-i n \phi}\right) .
$$

The translations $z \rightarrow z+d$ now act by

$$
\left(A_{m}, A_{n}, A_{-m}, A_{-n}\right) \rightarrow e^{i k d}\left(A_{m}, A_{n}, A_{-m}, A_{-n}\right),
$$

while the reflection $z \rightarrow-z$ acts by

$$
\left(A_{m}, A_{n}, A_{-m}, A_{-n}\right) \rightarrow\left(\bar{A}_{-m}, \bar{A}_{-n}, \bar{A}_{m}, \bar{A}_{n}\right) .
$$

Finally, the rotations $\phi \rightarrow \phi+\theta$ act by

$$
\left(A_{m}, A_{n}, A_{-m}, A_{-n}\right) \rightarrow\left(e^{i m \theta} A_{m}, e^{i n \theta} A_{n}, e^{-i m \theta} A_{-m}, e^{-i n \theta} A_{-n}\right) .
$$

In addition, in normal form, the vector field will commute with the normal form symmetry

$$
\left(A_{m}, A_{n}, A_{-m}, A_{-n}\right) \rightarrow\left(e^{i \omega \tau} A_{m}, e^{i \omega \tau} A_{n}, e^{-i \omega \tau} A_{-m}, e^{-i \omega \tau} A_{-n}\right)
$$

generated by phase shifts $t \rightarrow t+\tau$. The most general Hamiltonian vector field commuting with these operations, truncated at third order, is

$$
\begin{aligned}
\frac{d A_{m}}{d t} & =i \omega A_{m}+i A_{m}\left(s_{11}\left|A_{m}\right|^{2}+s_{12}\left|A_{n}\right|^{2}+s_{13}\left|A_{-m}\right|^{2}+s_{14}\left|A_{-n}\right|^{2}\right)+i r_{1} A_{n} A_{-n} \bar{A}_{-m} \\
\frac{d A_{n}}{d t} & =i \omega A_{n}+i A_{n}\left(s_{21}\left|A_{m}\right|^{2}+s_{22}\left|A_{n}\right|^{2}+s_{23}\left|A_{-m}\right|^{2}+s_{24}\left|A_{-n}\right|^{2}\right)+i r_{2} A_{m} A_{-m} \bar{A}_{-n} \\
\frac{d A_{-m}}{d t} & =-i \omega A_{-m}-i A_{-m}\left(s_{13}\left|A_{m}\right|^{2}+s_{14}\left|A_{n}\right|^{2}+s_{11}\left|A_{-m}\right|^{2}+s_{12}\left|A_{-n}\right|^{2}\right)-i r_{1} A_{n} A_{-n} \bar{A}_{m} \\
\frac{d A_{-n}}{d t} & =-i \omega A_{-n}-i A_{-n}\left(s_{23}\left|A_{m}\right|^{2}+s_{24}\left|A_{n}\right|^{2}+s_{21}\left|A_{-m}\right|^{2}+s_{22}\left|A_{-n}\right|^{2}\right)-i r_{2} A_{m} A_{-m} \bar{A}_{n} .
\end{aligned}
$$

With detuning and the symmetry breaking terms of the form $e^{i(m-n) \phi}$ we now obtain

$$
\frac{d A_{m}}{d t}=i \omega_{1} A_{m}+i \epsilon p A_{n}+i A_{m}\left(s_{11}\left|A_{m}\right|^{2}+s_{12}\left|A_{n}\right|^{2}+s_{13}\left|A_{-m}\right|^{2}+s_{14}\left|A_{-n}\right|^{2}\right)+i r_{1} A_{n} A_{-n} \bar{A}_{-m}
$$




$$
\begin{aligned}
\frac{d A_{n}}{d t} & =i \omega_{2} A_{n}+i \epsilon q A_{m}+i A_{n}\left(s_{21}\left|A_{m}\right|^{2}+s_{22}\left|A_{n}\right|^{2}+s_{23}\left|A_{-m}\right|^{2}+s_{24}\left|A_{-n}\right|^{2}\right)+i r_{2} A_{m} A_{-m} \bar{A}_{-n} \\
\frac{d A_{-m}}{d t} & =-i \omega_{1} A_{-m}-i \epsilon p A_{-n}-i A_{-m}\left(s_{13}\left|A_{m}\right|^{2}+s_{14}\left|A_{n}\right|^{2}+s_{11}\left|A_{-m}\right|^{2}+s_{12}\left|A_{-n}\right|^{2}\right)-i r_{1} A_{n} A_{-n} \bar{A}_{m} \\
\frac{d A_{-n}}{d t} & =-i \omega_{2} A_{-n}-i \epsilon q A_{-m}-i A_{-n}\left(s_{23}\left|A_{m}\right|^{2}+s_{24}\left|A_{n}\right|^{2}+s_{21}\left|A_{-m}\right|^{2}+s_{22}\left|A_{-n}\right|^{2}\right)-i r_{2} A_{m} A_{-m} \bar{A}_{n} .
\end{aligned}
$$

The equations corresponding to the perturbation $e^{i(m+n) \phi}$ can be obtained from those above by replacing $n$ with $-n$. Note that the two sets of equations differ in the nonlinear terms, since the change of sign of $n$ is not in general a symmetry of the $S O(2) \times O(2)$ equivariant problem. The Hamiltonian structure of these equations is the same as that described in $\S 2.8$.

The corresponding equations for the $S O(2) \times S O(2)$ interaction can be obtained from those above by setting $A_{-m}$ and $A_{-n}$ equal to zero (cf. equations (??)). Moreover setting $A_{n}$ and $A_{-n}$ equal to zero results in equations of the form (11). Note that the structure of these generalizations does not differ from the special cases already considered. Consequently no further analysis is necessary. This is because of the translation invariance in the axial direction which prevents terms that are resonant in the azimuthal coordinate from appearing in the normal form equations. Note also that we have restricted attention in the above discussion to the interaction between modes with the same axial wavenumbers only. It is not hard to generalize the discussion to cases where the competing modes have different axial wavenumbers as well.

\section{Amplitude equations for parametrically driven capillary waves and Benjamin-Feir instability}

\subsection{Parametrically driven capillary waves}

As already mentioned the equations derived above for the elliptical instability are the same as those arising in parametrically forced systems. In this section we discuss in more detail one such example: Milner's [1991] model for secondary instabilities in driven capillary waves. Milner is interested in understanding the relative stability between surface waves with roll and square planforms in a shallow layer of water contained in a large aspect ratio container oscillated vertically. In this configuration the dominant restoring force is due to surface tension, and the resulting surface ripples are called capillary waves. In the following we assume translation symmetry in two orthogonal directions, as well as reflection symmetry with respect to both directions. We write the eigenfunction describing the elevation of the surface relative to the oscillating undeformed surface in the form

$$
\zeta(x, y, t)=\operatorname{Re}\left\{e^{i \omega t}\left(v_{1} e^{i k x}+v_{2} e^{i k y}+w_{1} e^{-i k x}+w_{2} e^{-i k y}\right)\right\} .
$$

The translation symmetry $(x, y) \rightarrow\left(x+d_{1}, y+d_{2}\right)$ acts by

$$
\left(v_{1}, v_{2}, w_{1}, w_{2}\right) \rightarrow\left(e^{i k d_{1}} v_{1}, e^{i k d_{2}} v_{2}, e^{-i k d_{1}} w_{1}, e^{-i k d_{2}} w_{2}\right) ;
$$

reflection $(x, y) \rightarrow(x,-y)$ acts by

$$
\left(v_{1}, v_{2}, w_{1}, w_{2}\right) \rightarrow\left(v_{1}, w_{2}, w_{1}, v_{2}\right),
$$

while rotation through $\pi / 2$ acts by

$$
\left(v_{1}, v_{2}, w_{1}, w_{2}\right) \rightarrow\left(w_{2}, v_{1}, v_{2}, w_{1}\right)
$$


Finally, the normal form symmetry acts by $\left(v_{1}, v_{2}, w_{1}, w_{2}\right) \rightarrow e^{i \phi}\left(v_{1}, v_{2}, w_{1}, w_{2}\right)$. The resulting normal form equations, truncated at third order, take the Hamiltonian form (Milner [1991], Silber and Knobloch [1991])

$$
\begin{aligned}
& \frac{d v_{1}}{d t}=i \lambda v_{1}+i \epsilon \bar{w}_{1}+i v_{1}\left(a\left|w_{1}\right|^{2}+b\left(\left|v_{1}\right|^{2}+\left|w_{1}\right|^{2}\right)+c\left(\left|v_{2}\right|^{2}+\left|w_{2}\right|^{2}\right)\right)+i d v_{2} w_{2} \bar{w}_{1} \\
& \frac{d v_{2}}{d t}=i \lambda v_{2}+i \epsilon \bar{w}_{2}+i v_{2}\left(a\left|w_{2}\right|^{2}+b\left(\left|v_{2}\right|^{2}+\left|w_{2}\right|^{2}\right)+c\left(\left|v_{1}\right|^{2}+\left|w_{1}\right|^{2}\right)\right)+i d v_{1} w_{1} \bar{w}_{2} \\
& \frac{d w_{1}}{d t}=i \lambda w_{1}+i \epsilon \bar{v}_{1}+i w_{1}\left(a\left|v_{1}\right|^{2}+b\left(\left|v_{1}\right|^{2}+\left|w_{1}\right|^{2}\right)+c\left(\left|v_{2}\right|^{2}+\left|w_{2}\right|^{2}\right)\right)+i d v_{2} w_{2} \bar{v}_{1} \\
& \frac{d w_{2}}{d t}=i \lambda w_{2}+i \epsilon \bar{v}_{2}+i w_{2}\left(a\left|v_{2}\right|^{2}+b\left(\left|v_{2}\right|^{2}+\left|w_{2}\right|^{2}\right)+c\left(\left|v_{1}\right|^{2}+\left|w_{1}\right|^{2}\right)\right)+i d v_{1} w_{1} \bar{v}_{2}
\end{aligned}
$$

cf. equation (??). Here $\epsilon$ measures the amplitude of the parametric forcing and is responsible for the breaking of the normal form symmetry $\left(v_{1}, v_{2}, w_{1}, w_{2}\right) \rightarrow e^{i \phi}\left(v_{1}, v_{2}, w_{1}, w_{2}\right)$. The parametric forcing respects the spatial symmetries. The quantity $\lambda$ is the frequency mismatch, $\lambda \equiv \omega-\frac{1}{2} \omega_{d}$, where $\omega_{d}$ is the forcing frequency. Here $\omega$ is the natural oscillation frequency of the capillary waves.

The equations in the invariant subspace $v_{2}=w_{2}=0$ take the form

$$
\begin{aligned}
\frac{d v_{1}}{d t} & =i \lambda v_{1}+i \epsilon \bar{w}_{1}+i v_{1}\left(a\left|w_{1}\right|^{2}+b\left(\left|v_{1}\right|^{2}+\left|w_{1}\right|^{2}\right)\right) \\
\frac{d w_{1}}{d t} & =i \lambda w_{1}+i \epsilon \bar{v}_{1}+i w_{1}\left(a\left|v_{1}\right|^{2}+b\left(\left|v_{1}\right|^{2}+\left|w_{1}\right|^{2}\right)\right) .
\end{aligned}
$$

In the dissipative case these are the equations studied by Riecke et al. [1988] and Walgraef [1988]; they describe the effect of parametric forcing on the competition between standing and traveling waves. There is another important invariant subspace of equations (38), given by $v_{1}=w_{1}, v_{2}=$ $w_{2}$. This subspace corresponds to standing waves in the two orthogonal directions. Consequently traveling wave perturbations are suppressed. In this subspace the equations take the form

$$
\begin{aligned}
& \frac{d v_{1}}{d t}=i \lambda v_{1}+i \epsilon \bar{v}_{1}+i v_{1}\left(a\left|v_{1}\right|^{2}+b\left|v_{2}\right|^{2}\right)+i d v_{2}^{2} \bar{v}_{1}, \\
& \frac{d v_{2}}{d t}=i \lambda v_{2}+i \epsilon \bar{v}_{2}+i v_{2}\left(b\left|v_{1}\right|^{2}+a\left|v_{2}\right|^{2}\right)+i d v_{1}^{2} \bar{v}_{2} .
\end{aligned}
$$

These equations describe the parametric resonance in small aspect ratio square containers and have been studied by a number of authors (e.g., Nagata [1989]). Nearly square containers may be studied by breaking weakly the symmetry $\left(v_{1}, v_{2}\right) \rightarrow\left(v_{2}, v_{1}\right)$ in equations (40) (cf. Feng and Sethna [1989, 1990]; Feng and Wiggins [1993]). In a rectangular container the required mode interaction problem is of codimension two since the frequencies of the two competing modes must be tuned in order to resonate with half the frequency of the parametric forcing. Such an interaction is described by the equations

$$
\begin{aligned}
& \frac{d v_{1}}{d t}=i \lambda_{1} v_{1}+i \epsilon p_{1} \bar{v}_{1}+i v_{1}\left(s_{11}\left|v_{1}\right|^{2}+s_{12}\left|v_{2}\right|^{2}\right)+i d_{1} v_{2}^{2} \bar{v}_{1} \\
& \frac{d v_{2}}{d t}=i \lambda_{2} v_{2}+i \epsilon p_{2} \bar{v}_{2}+i v_{2}\left(s_{21}\left|v_{1}\right|^{2}+s_{22}\left|v_{2}\right|^{2}\right)+i d_{2} v_{1}^{2} \bar{v}_{2}
\end{aligned}
$$


with $\lambda_{1}-\lambda_{2}=O(1)$ and similarly for the remaining coefficients. Note that no transformation of the type used to simplify equations (11) is available in this case. As in our earlier discussions, the above Hamiltonian equations have two sets of integrals corresponding to the energy and, when there is an $S^{1}$ symmetry present, a conserved momentum. In particular, the system (39) is completely integrable while (40) and (41) are completely integrable if $\epsilon=0$. For example, equations (41) with $\epsilon=0$, has the integrals

$$
J=d_{2} r_{1}^{2}+d_{1} r_{2}^{2}, \quad E=\frac{\lambda_{1}}{d_{1}} r_{1}^{2}+\frac{\lambda_{2}}{d_{2}} r_{2}^{2}+r_{1}^{2} r_{2}^{2} \cos \phi+\frac{s_{11}-s_{21}}{2 d_{1}} r_{1}^{4}+\frac{s_{22}-s_{12}}{2 d_{2}} r_{2}^{4},
$$

where we have used the polar coordinate notation $v_{1}=r_{1} e^{i \phi_{1}}, v_{2}=r_{2} e^{i \phi_{2}}$ and defined $\phi \equiv 2 \phi_{1}-2 \phi_{2}$. (As before, one adjusts the coefficients in (41) without changing the reduced system to get a standard Hamiltonian system). A special case of these integrals was found already by Feng and Sethna [1990]. The existence of these integrals should prove helpful in analyzing the parametrically forced problem (41) for $0<\epsilon \ll 1$, as in the work of Feng and Sethna [1990] and Feng and Wiggins [1993], as should those obtaining in the case $\epsilon \neq 0, d_{1}=d_{2}=0$, discussed in $\S 3$. We also remark that there are hidden symmetries in the above problems, particularly in the square case (Crawford [1992]). These are relevant in the Hamiltonian case since Neumann boundary conditions have to be imposed at the boundaries of the container.

\subsection{Benjamin-Feir instability}

In the example discussed above, the parametric instability arose in the standard way, i.e., by temporal modulation of a parameter of the system, in this case the gravitational acceleration. There is, however, another example of parametric instability in the theory of water waves that also fits into our picture. This is the so-called Benjamin-Feir instability of wavetrains. This is a modulational instability of the wavetrain and arises through the coupling of two sidebands, $k \pm l$, via the wavenumber $k$ of the wavetrain. Two cases are of interest, that in which the original wavetrain is a progressive wavetrain, and that in which the original wavetrain is a standing wave. These two cases differ by the presence of a reflection symmetry in vertical planes in the latter case. This instability may be viewed as follows: the undisturbed water surface plays the role of the basic state corresponding to the flow with circular streamlines. The wavetrain then provides the distortion that can couple two natural modes of oscillation of the system leading to the possibility of subharmonic instabilities of Benjamin-Feir type.

We illustrate the above discussion with the nonlinear Schrödinger equation

$$
\frac{\partial A}{\partial t}=i \gamma \frac{\partial^{2} A}{\partial x^{2}}+i|A|^{2} A
$$

subject to periodic boundary conditions in the spatial variable $x$. Equation (43) has a solution in the form of a wave $A=R e^{i \Omega t+i k x}$, where $\Omega=R^{2}-\gamma k^{2}$. We wish to study the stability of this solution with respect to side band perturbations, i.e., with respect to perturbations with wavenumbers $k \pm l$. Thus we set

$$
A=e^{i \Omega t}\left(R e^{i k x}+a\right)
$$

Linearizing in $a$, we find that $a$ satisfies the equation

$$
\frac{\partial a}{\partial t}=i \gamma \frac{\partial^{2} a}{\partial x^{2}}+i\left(2 R^{2}-\Omega\right) a+i R^{2} e^{2 i k x} \bar{a}
$$


This equation has a solution of the form

$$
a=b_{1} e^{i(k+l) x}+b_{2} e^{i(k-l) x},
$$

where

$$
\begin{aligned}
& \frac{d b_{1}}{d t}=i\left[2 R^{2}-\Omega-\gamma(k+l)^{2}\right] b_{1}+i R^{2} \bar{b}_{2} \\
& \frac{d b_{2}}{d t}=i\left[2 R^{2}-\Omega-\gamma(k-l)^{2}\right] b_{2}+i R^{2} \bar{b}_{1} .
\end{aligned}
$$

These equations are of the form (21), with $\lambda_{1}-\lambda_{2}=O(l), p_{1}=p_{2}=-1$, and $R^{2}$ playing the role of $\epsilon$. Condition (22) implies that an instability is present whenever

$$
0<\gamma\left(2 R^{2}-\gamma l^{2}\right)
$$

Consequently the wavetrain is unstable with respect to sideband instability $(0<l \ll 1)$ whenever $\gamma>0$. This is the Benjamin-Feir instability (cf. Benjamin [1967]).

In this discussion we have focused on the linear stability properties of a propagating wavetrain. To determine the nonlinear terms responsible for the saturation of the instability one would have to go through a center manifold type of reduction based on the two unstable modes. The structure of these terms is not as simple as in (21); this is because in the above derivation we are considering an instability of a wave, and this wave has already broken the $O(2)$ symmetry of the system. On the other hand if we consider the two sidebands, $k \pm l$, as two modes that are (weakly) coupled by a small amplitude wave, then to leading order (in $R$ ) the equations for $b_{1}$ and $b_{2}$ will be of the form

$$
\begin{aligned}
& \frac{d b_{1}}{d t}=i R^{2} \bar{b}_{2}+i g_{11} b_{1}+i g_{12} \bar{b}_{1}^{k-l-1} b_{2}^{k+l} \\
& \frac{d b_{2}}{d t}=i R^{2} \bar{b}_{1}+i g_{21} b_{2}+i g_{22} \bar{b}_{2}^{k+l-1} b_{1}^{k-l},
\end{aligned}
$$

where the functions $g_{i j}, i=1,2, j=1,2$, are functions of

$$
\left|b_{1}\right|^{2},\left|b_{2}\right|^{2}, b_{1}^{k-l} \bar{b}_{2}^{k+l}+\bar{b}_{1}^{k-l} b_{2}^{k+l} \text { and }\left(b_{1}^{k-l} \bar{b}_{2}^{k+l}-\bar{b}_{1}^{k-l} b_{2}^{k+l}\right)^{2},
$$

as well as of $R$. For $k>2, l \geq 1, k>l$, the resulting equations truncated at third order are precisely of the form (11), though with broken reflection symmetry $\left(b_{1} \leftrightarrow b_{2}\right)$, and so can be transformed into equations (21). The Benjamin-Feir instability is thus of the same kind as the instabilities discussed here. We remark, finally, that the instability of standing wavetrains in dispersive systems is complicated by the finite group velocity of the waves, and so is described by equations that are more complicated than (43) (see Knobloch [1992a]).

\section{Precessional instability of columnar flows: an explicit ex- ample}

In this section we discuss an explicit application of the above ideas. We focus on the precessional instability of columnar flows of the form $(0, V(r), W(r))$, where $(r, \phi, z)$ are right-handed cylindrical coordinates. Szeri and Holmes [1988] have established sufficient conditions for the nonlinear stability

of such flows to finite amplitude axisymmetric disturbances using the energy-Casimir method. The 
method depends upon finding a constant of motion that has a local maximum or minimum at the corresponding equilibrium. In general such a constant of motion is a functional of the kinetic energy and the conserved quantities that correspond to symmetries of the system via Noether's theorem. The nonlinear stability to axisymmetric perturbations is proved by showing that the second variation of this functional is positive (negative) definite. We show that the energy-Casimir functional becomes indefinite if three-dimensional variations are allowed. The idea of the proof is as follows. We assume that an infinitesimal external Coriolis force is applied to the system. As a result the system loses some of its conserved quantities (e.g. angular momentum about the $z$ axis). In addition the Coriolis force alters the base flow. We show that the resulting steady state flow has an unstable manifold for an arbitrarily small strength of the external Coriolis force, and conclude that columnar flows are structurally unstable in the sense that they are infinitesimally close to flows (steady state solutions of Euler equations) having an unstable manifold. We formalize this discussion by making the following definition: A steady-state solution $V_{0}$ of a Hamiltonian system with a Hamiltonian $H_{0}$ is called structurally unstable if for any $\epsilon_{0}>0$ there exists an $\epsilon, 0<\epsilon<\epsilon_{0}$, such that the steady state $V_{0}$ is deformed into a steady state solution $V_{\epsilon}=V_{0}+\epsilon V_{1}$ of a Hamiltonian system with a Hamiltonian $H_{\epsilon}=H_{0}+\epsilon H_{1}$ having an unstable manifold.

We remark that any velocity field of the form $(0, V(r), W(r))$ satisfies the Euler equations for the fluid regardless of the functions $V(r)$ and $W(r)$. The linear stability of these flows to axisymmetric perturbations was first considered by Rayleigh, who found that the flow $(0, V(r), 0)$ is stable only if

$$
\Phi=r^{-3} \frac{d}{d r}\left(r^{2} V^{2}(r)\right)>0
$$

for all $r$ in the domain of interest. Synge [1933] showed that $\Phi>0$ is necessary and sufficient for stability. Howard and Gupta [1962] derived a sufficient condition for linear stability of the flow with velocity $(0, V(r), W(r))$, including an axial velocity component $W$. The condition is

$$
J=\Phi /(d W / d r)^{2}>1 / 4 .
$$

Thus if the Richardson number $J$ exceeds $1 / 4$ everywhere in the domain of interest, then the flow is linearly stable.

As already noted the two cases $W=0$ and $W \neq 0$ possess different symmetry properties with respect to reflections $z \rightarrow-z$. The former has the symmetry $O(2) \times S O(2)$, while the latter has the symmetry $S O(2) \times S O(2)$. This distinction is important for the structure of the full problem (compare $\S 2.1$ and $\S 2.9$ ), but does not affect the linear stability calculation, i.e.,, the calculation of the quantity $p q$ that distinguishes splitting from passing. In the following we restrict ourselves to the reflection symmetric flows $(0, V(r), 0)$ satisfying the inviscid Rayleigh criterion for stability. The results of our analysis are presented for flows selected from the two-parameter family of Burger vortices given by

$$
V(r)=\frac{\Gamma}{2 \pi r}\left(1-e^{-\beta r^{2}}\right) .
$$

These profiles are of interest in the vortex breakdown problem. The results discussed below focus on the presence of splitting and hence of instability; no nonlinear computations have been carried out.

The flow configuration is shown in Figure 5 . The $\mathbf{E}$ axis is the axis of rotation for the system. The unperturbed flow velocity field is given by (52). In a coordinate system rotating with a constant angular velocity about $\mathbf{E}$, the inviscid Euler equations require the instantaneous velocity field to satisfy

$$
\frac{\partial \mathbf{v}}{\partial t}+\mathbf{v} \cdot \nabla \mathbf{v}+2 \epsilon \mathbf{E} \times \mathbf{v}=-\nabla \pi
$$


where $\mathbf{E}=(\cos \phi,-\sin \phi, 0)$. Here $\epsilon$ is the strength of the external Coriolis force. For $\epsilon=0$ we find that any flow $(0, V(r), 0)$ satisfies $(53)$. In the presence of the external Coriolis force the following is an exact solution of the inviscid Euler equations (53):

$$
\mathbf{u}_{0}=(0, V(r),-2 \epsilon r \sin \phi), \quad \pi_{0}=\int V^{2} / r d r-2 \epsilon^{2} r^{2} \sin ^{2} \phi .
$$

We now consider the linear stability analysis of this flow. The linearized equations for the disturbance field $(u, v, w, p)$ are

$$
\begin{aligned}
\frac{\partial u}{\partial t}+\frac{V}{r} \frac{\partial u}{\partial \phi}-2 \frac{V}{r} v-2 \epsilon r \sin \phi \frac{\partial u}{\partial z}-2 \epsilon w \sin \phi & =-\frac{\partial p}{\partial r} \\
\frac{\partial v}{\partial t}+\frac{V}{r} \frac{\partial v}{\partial \phi}+\left(\frac{V}{r}+V^{\prime}(r)\right) u-2 \epsilon r \sin \phi \frac{\partial v}{\partial z}-2 \epsilon w \cos \phi & =-\frac{1}{r} \frac{\partial p}{\partial \phi} \\
\frac{\partial w}{\partial t}+\frac{V}{r} \frac{\partial w}{\partial \phi}-2 \epsilon r \sin \phi \frac{\partial w}{\partial z} & =-\frac{\partial p}{\partial z} \\
\frac{1}{r} \frac{\partial}{\partial r}(r u)+\frac{1}{r} \frac{\partial v}{\partial \phi}+\frac{\partial w}{\partial z} & =0 .
\end{aligned}
$$

Dispersion curves for the Burger's vortex (52) with $\frac{\Gamma}{2 \pi}=1$ and $\beta=1$ are shown in Figure 6 . The solid and dashed curves correspond to axisymmetric $(m=0)$ and helical $(m=1)$ modes. For $\epsilon=0$ the system (55) has invariant subspaces characterized by different azimuthal wavenumbers. Perturbations with $\epsilon \neq 0$ couple these subspaces. As a result the movement of the eigenvalues changes from passing to splitting provided the azimuthal wavenumbers differ by 1 (Figure 7 ). We can associate vortex instabilities with the degeneracies (crossing points in Figure 6) caused by two physically distinguishable eigenmodes of the unperturbed vortex having the same eigenfrequency.

\section{Discussion}

In many problems stability results can be obtained from variational principles using the available conserved quantities (momentum maps or Casimir functions). As in the example of columnar flows above such a variational formulation exists for many exact solutions in two-dimensional hydrodynamics and plasma physics, as well as for more general Hamiltonian systems, and allows one to use the conserved quantities to establish the nonlinear stability of such equilibria. The situation is less clear in three-dimensional hydrodynamics. In some cases, however, it is possible to use the conserved quantities obtained via Noether's theorem to prove that an equilibrium must be linearly unstable. If the system is distorted (perturbed) in such a way that at least one conserved quantity is lost, its evolution satisfies equations of the form

$$
\frac{d}{d t} F(u)=\epsilon G(u)
$$

where $F(u)$ is a conserved quantity (quantities) for the undistorted system (obtained via Noether's theorem) and $G(u)$ is a functional describing the rate of loss of the conserved quantity (quantities) $F(u)$. Here $\epsilon$ is the strength of the system symmetry breaking perturbation. Although equations (56) are fully nonlinear, their linearization about $u=0$ is given by

$$
\left\langle L_{F}, \frac{d u}{d t}\right\rangle=\epsilon\left\langle L_{G}, u\right\rangle
$$


where $L_{F}=D F(0)$ and $L_{G}=D G(0)$ are two linear operators. Equation (57) is valid under the assumption that $\|u\|$ is small. In the case in which the distortion leads to a parametric instability involving two critical modes $u_{1}$ and $u_{2}$,

$$
\frac{d A_{1}}{d t}=\epsilon p A_{2}, \quad \frac{d A_{2}}{d t}=\epsilon q A_{1}
$$

where $A_{1}$ and $A_{2}$ are the corresponding amplitudes. It follows that instability is present if $p q>0$, with a growth rate given by

$$
\sigma^{2}=\epsilon^{2} p q
$$

An expression for $\sigma^{2}$ can be found in terms of the critical modes and $L_{F}, L_{G}$ only. Since

$$
u=A_{1}(t) u_{1}+A_{2}(t) u_{2}
$$

it follows that

$$
p\left\langle L_{F}, u_{1}\right\rangle A_{2}+q\left\langle L_{F}, u_{2}\right\rangle A_{1}=\left\langle L_{G}, u_{1}\right\rangle A_{1}+\left\langle L_{G}, u_{2}\right\rangle A_{2}
$$

and hence that

$$
p\left\langle L_{F}, u_{1}\right\rangle=\left\langle L_{G}, u_{2}\right\rangle, \quad q\left\langle L_{F}, u_{2}\right\rangle=\left\langle L_{G}, u_{1}\right\rangle .
$$

Finally, therefore,

$$
\sigma^{2}=\epsilon^{2} \frac{\left\langle L_{G}, u_{1}\right\rangle\left\langle L_{G}, u_{2}\right\rangle}{\left\langle L_{F}, u_{1}\right\rangle\left\langle L_{F}, u_{2}\right\rangle} .
$$

If the quantity (63) is positive the equilibrium is linearly unstable. The formula (63) may be viewed as an analogue of the formula for the movement of eigenvalues in the context of dissipation induced instabilities (see Bloch et al. [1993]).

The above discussion illustrates well the basic point of this paper: that system symmetry breaking perturbations of Hamiltonian systems with symmetry can, under the appropriate circumstances, lead to the loss of stability. These instabilities take place whenever the loss of symmetry results in the splitting of double eigenvalues and are important in applications since they occur on a dynamical time scale. This is so, for example, for the elliptical instability of columnar flow (Pierrehumbert [1986], Bayly [1986]). As discussed here (see also Guckenheimer and Mahalov [1992]) the origin of this instability is universal. It requires only the presence of reflection symmetry in the axial direction. If the two modes are interchanged by this symmetry, then the coefficients of the $S O(2)$-breaking terms must be equal i.e., $p=q$ in (11), and so $p q>0$ implying splitting. This argument establishes the existence of an instability without the necessity of having to carry out even the linear stability calculation. In this paper we have extended this approach to other multiple eigenvalues, and in particular considered the case of the Hamiltonian Hopf bifurcation, with or without an additional (axial) reflection symmetry. In this case when the system symmetry is broken, the eigenvalues may either split or bounce, indicating the need for a linear stability analysis. In addition we have shown how the symmetries of the system can be used to write down the truncated normal forms describing the growth and saturation of these instabilities. We have described the Hamiltonian structure of the resulting normal forms, and showed that in the simplest cases of interest these normal forms are completely integrable. As a result a complete description of the local dynamics becomes possible.

We focused on systems with the symmetries $S O(2) \times S O(2)$ or $O(2) \times S O(2)$, where the first group refers to periodic boundary conditions in the axial direction and the second to rotational invariance. For such systems instabilities of the type discussed here are expected to be always present since 
the translational invariance in the axial direction implies that the axial wavenumber of the modes is available as a parameter that can be tuned to force the coalescence of dangerous eigenmodes on the imaginary axis. The dangerous interactions are precisely those for which independent passing does take place in the unperturbed problem (cf. Dellnitz et al. [1992]). As illustrated in Figure 8, once the azimuthal $S O(2)$ symmetry is reduced by the system symmetry breaking perturbation the bifurcations that take place as a function of the detuning $\lambda$ are now non-semisimple double Hopf bifurcations with $1: 1$ resonance (cf. van Gils et al. [1990]).

Acknowledgements We thank Mary Silber and Vivien Kirk for helpful discussions on the Hamiltonian structure of normal forms.

\section{Bibliography}

D. Armbruster and A. Mahalov [1992], On the explicit symmetry breaking in the Taylor-Couette problem, Phys. Lett. A, 167, 251-254.

B.J. Bayly [1986], Three-dimensional instability of elliptical flow, Phys. Rev. Lett., 57, 2160-2163.

T.B. Benjamin [1967], Instability of periodic wavetrains in nonlinear dispersive systems, Proc. Roy. Soc. London A, 299, 59-75.

A.M. Bloch, P.S. Krishnaprasad, J.E. Marsden and T.S. Ratiu [1993], Dissipation-induced instabilities, preprint.

J.D. Crawford [1992], Normal forms for driven surface waves: boundary conditions, symmetry and genericity, Physica D, 52, 429-457.

R. Cushman and D.L. Rod [1982], Reduction of the semisimple 1: 1 resonance, Physica D, 6, $105-112$.

planar vector fields,

G. Dangelmayr and E. Knobloch [1991], Hopf bifurcation with broken circular symmetry, Nonlinearity, 4, 399-427.

M. Dellnitz, I. Melbourne and J.E. Marsden [1992], Generic bifurcation of Hamiltonian vector fields with symmetry, Nonlinearity, 5, 979-996.

Z.C. Feng and P.R. Sethna [1989], Symmetry breaking bifurcations in resonant surface waves, J. Fluid Mech., 199, 495-518.

Z.C. Feng and P.R. Sethna [1990], Global bifurcations and chaos in parametrically forced systems with one-one resonance, Dyn. Stab. Systems, 5, 201-225.

Z.C. Feng and S. Wiggins [1993], On the existence of chaos in a class of two-degree-of-freedom, damped, strongly parametrically forced mechanical systems with broken $O(2)$ symmetry, $Z$. angew. Math. Phys., 44, 201-248.

D.M. Galin [1982], Versal deformations of linear Hamiltonian systems, AMS Transl. 118, 1-12, (Trudy Sem. Petrovsk. 1, 63-74, 1975).

S.A. van Gils, M. Krupa and W.F. Langford [1990], Hopf bifurcation with non-semisimple $1: 1$ resonance, Nonlinearity, 3, 825-850. 
J. Guckenheimer and A. Mahalov [1992], Instability induced by symmetry reduction, Phys. Rev. Lett., 68, 2257-2260.

G. Haller and S. Wiggins [1992], Geometry and chaos near resonant equilibria of three degree of freedom Hamiltonian systems, preprint.

G. Haller and S. Wiggins [1993], Orbits homoclinic to resonances: the Hamiltonian case, Physica $D, 66,298-346$.

L.N. Howard and A.S. Gupta [1962], On the hydrodynamic and hydromagnetic stability of swirling flows, J. Fluid Mech., 14, 463-476.

T. Iwai [1985], On reduction of two degrees of freedom Hamiltonian systems by an $S^{1}$ action, and $S O_{0}[1,2]$ as a dynamical group, J. Math. Phys., 26, 885-893.

M.F. Jorgensen and P.L. Christiansen [1992], Hamiltonian structure for a modified discrete selftrapping dimer, preprint.

R.R. Kerswell [1992], Instabilities of tidally and precesionally induced flows, preprint.

E. Knobloch [1992a], Nonlocal amplitude equations, in Pattern Formation in Complex Dissipative Systems, S. Kai, ed., World Scientific, 263-274.

E. Knobloch [1992b], Bifurcations in rotating systems, in Theory of Solar and Planetary Dynamos: Introductory Lectures, M.R.E. Proctor and A.D. Gilbert, eds., Cambridge University Press, in press.

E. Knobloch and M. Silber [1993], Oscillatory convection in a rotating layer, Physica D, 63, 213232.

M.G. Krein [1950], A generalization of several investigations of A.M. Liapunov on linear differential equations with periodic coefficients, Dokl. Akad. Nauk SSSR, 73, 445-448.

M. Kummer [1979] On resonant Hamiltonian systems with finitely many degrees of freedom, Springer Lecture Notes in Physics 93, 57-75.

M. Kummer [1990], On resonant classical Hamiltonians with $n$ frequencies, J. Diff. Eq., 83, 220243.

S. Leibovich, S.N. Brown and Y. Patel [1986], Bending waves on inviscid columnar vortices, J. Fluid Mech., 173, 595-624.

D. Lewis and J.E. Marsden [1989] The Hamiltonian-dissipative decomposition of normal forms of vector fields. Proc of the conf. on Bifurcation Theory and its Numerical Analysis, Xi'an Jaitong Univ. Press, 51-78.

A. Mahalov [1993], The instability of rotating fluid columns subjected to a weak external Coriolis field, Phys. Fluids A, 5, 891-900.

J.E. Marsden [1992], Lectures on Mechanics, Cambridge University Press.

A. Mielke [1992] Hamiltonian and Lagrangian Flows on Center Manifolds, with Applications to Elliptic Variational Problems, Springer Lecture Notes in Mathematics 1489.

J. Miles [1991], Nonlinear asymmetric excitation of edge waves, Int. J. Appl. Math., 46, 101-108. 
S.T. Milner [1991], Square patterns and secondary instabilities in driven capillary waves, J. Fluid Mech., 225, 81-100.

W. Nagata [1988], Dynamics near a symmetric Hopf bifurcation, Proc. Roy. Soc. Edin., 108A, 249-267.

W. Nagata [1989], Nonlinear Faraday resonance in a box with a square base, J. Fluid Mech., 209, $265-284$.

R.T. Pierrehumbert [1986], Universal short-wave instability of 2-dimensional eddies in an inviscid fluid, Phys. Rev. Lett., 57, 2157-5159.

H. Riecke, J.D. Crawford and E. Knobloch [1988], Time-modulated oscillatory convection, Phys. Rev. Lett., 61, 1942-1945.

M. Silber and E. Knobloch [1991], Hopf bifurcation on a square lattice, Nonlinearity, 4, 1063-1106.

J.W Swift [1988], Hopf bifurcation with the symmetry of a square, Nonlinearity, 1, 333-377.

J.L. Synge [1933], The instability of heterogeneous fluids, Trans. R. Soc. Can., 27, 1-18.

A. Szeri and P. Holmes [1988], Nonlinear stability of axisymmetric swirling flows, Phil. Trans. R. Soc. Lond. A, 326, 327-354.

V.A. Vladimirov and K. Il'in [1988], Three-dimensional instability of an elliptical Kirchoff vortex, Fluid Dynamics, 3, 356-360.

F.A. Waleffe [1990], On the three-dimensional instability of a strained vortex, Phys. Fluids A, 2, $76-80$.

D. Walgraef [1988], External forcing of spatio-temporal patterns, Europhys. Lett., 7, 485-491.

\section{Figure Captions}

Figure 1. Bifurcation diagram for $m=1$ in the + case (splitting): (a) $c>1$, (b) $c<1$. The label $S$ denotes a stable equilibrium (center) while $U$ denotes an unstable equilibrium (saddle).

Figure 2. Bifurcation diagram for $m=1$ in the - case (passing). The label $S$ denotes a stable equilibrium (center) while $U$ denotes an unstable equilibrium (saddle).

Figure 3. The transition between splitting and passing for $m=1$, showing (a) $N(\lambda)$ and (b) $w(\lambda)$ when $q=0$. This case connects Figure $1 \mathrm{~b}$ with Figure 2 .

Figure 4. Elliptical instability $(m=2)$. Phase portrait for standing oscillations (a) $\lambda>\epsilon>0$, (b) $|\lambda|<\epsilon$, (c) $\lambda<-\epsilon$.

Figure 5. Coordinate system for the columnar vortex subjected to an external Coriolis force.

Figure 6. Dispersion curves for the Burger's vortex. The solid and dashed curves correspond to axisymmetric $(m=0)$ and helical $(m=1)$ modes.

Figure 7. Movement of eigenvalues: (a) independent passing $(\epsilon=0)$, (b) splitting $(\epsilon \neq 0)$.

Figure 8. Independent passing $(\epsilon=0)$ and non-semisimple Hamiltonian Hopf $(\epsilon \neq 0)$. 